\title{
Porous layer impedance applied to a moving wall: Application to the radiation of a covered piston
}

\author{
Olivier Doutres, ${ }^{\text {a) }}$ Nicolas Dauchez, and Jean-Michel Génevaux \\ Laboratoire d'Acoustique UMR CNRS 6613, Univ. du Maine, 72095 Le Mans cedex 9, France
}

(Received 27 June 2006; revised 6 September 2006; accepted 6 September 2006)

\begin{abstract}
Modeling a porous layer mounted on a vibrating structure using acoustic impedance is investigated in this paper. It is shown that the use of surface impedance usually measured with the impedance tube method can provide an inaccurate estimation of the acoustic pressure radiated by the covered structure. The paper focuses on the derivation of an impedance, denoted the "transfer impedance," which describes accurately the dynamic movement of the porous layer. Biot's theory is used in the model to account for deformations in the thickness of the layer. Experimental validation is performed using a circular piston covered by a foam or a fibrous layer, radiating in an infinite half space. The radiation model including the transfer impedance shows close agreement with experimental data. (C) 2007 Acoustical Society of America. [DOI: 10.1121/1.2359233]
\end{abstract}

PACS number(s): 43.50.Gf, 43.40.Rj, 43.20.Rz [KA] Pages: 206-213

\section{INTRODUCTION}

The reduction of the sound radiated by vibrating structures is usually performed using covering materials. If the structure is immersed in a heavy acoustic fluid, as in marine applications, the covering decouples the surrounding fluid from the vibrations of the structure due to deformation in the thickness. ${ }^{1-3}$ In the case of light surrounding fluid, as in building or transport applications (car, aircraft, train), porous materials such as polymer foams and fibrous materials are widely used because they provide sound absorption and radiation attenuation due to interaction between the porous frame and the saturating fluid. ${ }^{4}$

The behavior of a layer attached to a vibrating structure can be described by using a simple model based on an impedance formulation. This model, known as "locally reacting," assumes that the motion at a point of the covering surface is independent of the motion of any other point of the surface area. When considering immersion in an acoustically heavy fluid, ${ }^{5,6}$ this impedance is usually written in the form

$$
Z_{t}=\frac{p}{v_{p}-v},
$$

where $p$ is the pressure exerted by the fluid on the fluidcovering interface, $v$ the velocity of the same covering surface and $v_{p}$ is the velocity of the base plate (equal to the velocity on the other covering surface). In these studies, the covering is assumed to behave like massless distributed springs and the impedance is derived only by means of its thickness and bulk modulus. This simple model does not account for wave effects in the layer; however, it provides a good description of the main vibratory phenomena.

For acoustic radiation problems relating to vibrating structures immersed in light fluid, the impedance formulation is frequently used because of its apparent simplicity. How-

\footnotetext{
a) Author to whom correspondence should be addressed; electronic mail: olivier.doutres@univ-lemans.fr
}

ever, according to the authors' knowledge, a clear ambiguity appears regarding the definition of the impedance to be applied to the moving structure. For example, in their book, Morse and Ingard ${ }^{7}$ give an expression for the far field radiated pressure of a nonrigid boundary plane. The active and passive regions of the boundary plane are characterized by the same acoustic impedance which is defined as the structure reaction to an incoming pressure wave. This impedance is usually called the surface impedance and is given in the following form:

$$
Z_{s}=\frac{p}{v}
$$

where $p$ and $v$ are, respectively, the acoustic pressure and the particle velocity at the nonrigid boundary surface. Mangulis ${ }^{8,9}$ has already pointed out that the active and passive regions of a nonrigid plane should not be characterized by the same impedance. The case of a circular piston set in an infinite baffle was investigated, considering the characteristic impedances as vibratory impedances defined in terms of their mass per unit area. The same ambiguity in the definition of the acoustic impedance exists for applications dealing with porous coverings. Suzuki, Maruyama, and Ido, ${ }^{10}$ investigated the effect of porous coverings on the pressure radiated by vibrating structures in a vehicle cabin in terms of an acoustic impedance as in the form of Eq. (2). In the case of porous coverings, $p$ and $v$ of Eq. (2) are the pressure and the normal component of velocity in the free air close to the front face of the material. This impedance applied to a porous layer is generally experimental data which can be obtain using a common method such as an impedance tube. ${ }^{11}$ However, by using this impedance, the moving boundary condition on the vibrating surface is not taken into account. In the same way, Piot, Ville, and Auger ${ }^{12}$ modeled the behavior of an absorbent material in dynamic conditions using the surface impedance $Z_{s}$. More recently, Shao and Mechefske ${ }^{13}$ used a surface impedance independent of the frequency to account for absorbing materials mounted on vibrating cylindrical ducts, but, as in the previous models, the moving 
boundary condition is not clearly demonstrated in that case.

The problem considered in this paper focuses on the ambiguity of acoustic impedance applied to a porous material attached to a moving structure. An impedance as in Eq. (1), usually used for heavy fluid configurations, is implemented in the case of a covered boundary moving in a light acoustic fluid: the moving behavior of the covering is taken into account. The first part of the paper presents the acoustic boundary conditions linked to the impedances $Z_{s}$ and $Z_{t}$. Then, both impedances are derived for two kinds of porous material commonly encountered in industrial applications. Both materials differ by their mechanical properties: one is a relatively rigid foam layer and the other, a limp fibrous layer. A one-dimensional model based on Biot's theory ${ }^{14}$ is used to take into account wave propagation in the solid and fluid phases of the porous media. Using Biot's theory is of great importance for the moving boundary configuration because the frame of the porous layer is directly excited by the vibrating structure. In order to validate the porous modeling using $Z_{t}$ and illustrate the consequences of using $Z_{s}$ instead of $Z_{t}$ in an acoustic problem with moving covered boundaries, the derived impedances are applied to the radiation problem of a piston covered with the porous layers and set in an infinite baffle. Finally, these simulations are compared to acoustic radiation measurements.

\section{IMPEDANCE LINKED TO COVERED BOUNDARIES}

\section{A. Definition of the boundary conditions}

Let us consider an acoustic domain $\Omega$ limited by the nonrigid boundary $\Gamma=\partial \Omega$ as illustrated in Fig. 1. The normal to the boundary $\Gamma$, outwardly directed, is denoted $n$. The harmonic pressure field $p$ is governed by the homogeneous linear wave equation (the time factor $e^{j \omega t}$ is omitted throughout this paper),

$$
\nabla^{2} p+k^{2} p=0 \text { in } \Omega,
$$

where the wave number is $k=\omega / c_{0}$, with $\omega$ as the angular frequency and $c_{0}$ the speed of sound in the medium. In the case of an acoustic problem involving a porous covering, the boundary condition can be expressed in terms of a harmonic acoustic impedance. If the backing is a rigid and impervious wall [see Fig. 2(a)], the impedance applied to $\Gamma$, denoted in this paper as the "surface impedance" $Z_{s}$, is usually given as in Eq. (2). This impedance expresses the coupling between the scalar pressure and the $z$ component of particle velocity in the free air close to the front face of the porous layer, respectively $p(0)$ and $v(0)$. Substituting the normal particle velocity $v$ in Eq. (2) for the one-dimensional Euler equation

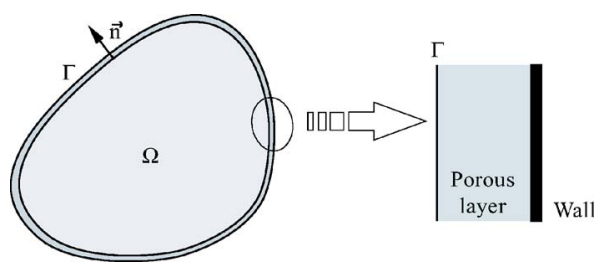

FIG. 1. System consisting of an acoustic domain $\Omega$ limited by a nonrigid boundary $\Gamma$.

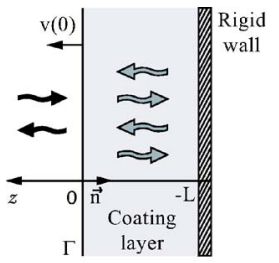

(a)

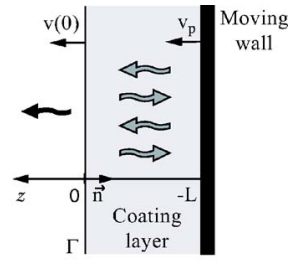

(b)
FIG. 2. Boundary condition: (a) static covered wall, (b) moving covered wall.

$$
v=-\frac{1}{j \omega \rho_{0}} \frac{\partial p}{\partial z},
$$

gives the associated boundary condition

$$
\frac{\partial p}{\partial z}+j k \beta_{s} p=0 \quad \text { on } \Gamma,
$$

where $\beta_{s}=\rho_{0} c_{0} / Z_{s}$, with $\rho_{0}$ as the acoustic fluid density. This boundary condition is known as the homogeneous mixed Dirichlet-Neumann boundary condition or Robin boundary condition. The acoustic impedance $Z_{s}$ to be implemented in this boundary condition is easily measured, using the impedance tube method, for example. In addition, this impedance is mainly used to derive the absorption coefficient of a porous layer backed by a rigid wall.

Let us now consider the covered wall moved by a harmonic velocity of magnitude $v_{p}$ [see Fig. 2(b)]. As usually encountered in literature dealing with covered plates immersed in heavy fluid, the impedance applied to the boundary can be written in terms of the relative velocity between the velocity of the wall $v_{p}$ and the normal component of the fluid velocity at the fluid-porous interface as written in Eq. (1). This formulation involves a transfer of energy from the moving wall to the acoustic medium through the covering and will thus be denoted in this paper as the "transfer impedance."

By substituting the normal particle velocity $v$ in Eq. (1) for Eq. (4), the associated boundary condition is written as an inhomogeneous mixed boundary condition

$$
-\frac{\partial p}{\partial z}+j k \beta_{t} p=j \omega \rho_{0} v_{p} \quad \text { on } \Gamma,
$$

where $\beta_{t}=\rho_{0} c_{0} / Z_{t}$. The source component on the right hand side of Eq. (6) appears because the impedance is expressed in terms of the wall velocity $v_{p}$. As a consequence, it may be difficult to measure the impedance, although it is usually considered as experimental data.

However, in most of the applications met in the literature which involve porous coverings, the acoustic impedance $Z_{s}$ characteristic of Eq. (5) is also used in Eq. (6) instead of $Z_{t}$. This may be explained by the fact that only $Z_{s}$ is easily obtained by experiment. However, substituting $Z_{t}$ for $Z_{s}$ may give an inaccurate estimation of the acoustic pressure generated in the domain $\Omega$. This is illustrated in the following, first, by deriving both impedances with a one-dimensional model based on Biot's theory, and then, by implementing these impedances in a radiation problem of a covered piston. 
TABLE I. Properties of the porous materials.

\begin{tabular}{lcc}
\hline \hline Porous & $\mathrm{A}$ & $\mathrm{B}$ \\
\hline Thickness: $L(m \mathrm{~m})$ & 18.9 & 29 \\
\hline Air flow resistivity: $\sigma\left(\mathrm{N} \mathrm{s} / \mathrm{m}^{4}\right)$ & 57000 & 105000 \\
Porosity: $\phi$ & 0.97 & 0.95 \\
Tortuosity: $\alpha_{\infty}$ & 1.54 & 1 \\
Viscous length: $\Lambda(\mu \mathrm{m})$ & 24.6 & 35.1 \\
Thermal length: $\Lambda^{\prime}(\mu \mathrm{m})$ & 73.8 & 105.3 \\
Skeleton density: $\rho_{1}\left(\mathrm{~kg} / \mathrm{m}^{3}\right)$ & 46 & 17 \\
Young's modulus at $5 \mathrm{~Hz}: E(\mathrm{kPa})$ & 214 & 1.4 \\
Structural loss factor at $5 \mathrm{~Hz}: \eta$ & 0.115 & 0.1 \\
Poisson's ratio: $\nu$ & 0.3 & 0 \\
\hline \hline
\end{tabular}

\section{B. Derivation of the acoustic impedance for a locally reacting porous material}

To derive the transfer impedance $Z_{t}$ [Eq. (1)], it is necessary to calculate the acoustic pressure and porous surface particle velocity induced by the wall movement $v_{p}$. As a result of the "locally reacting" assumption, a simple onedimensional model may be used. It assumes that only plane waves propagate in the $z$ direction. It is interesting to note that this model is equivalent to the transfer matrix method ${ }^{4,15}$ where the multilayer was excited by plane waves with normal incidence.

The theoretical one-dimensional model of the porous layer is based on Biot's theory ${ }^{4,14}$ to take into account vibration of both solid and fluid phases induced by the wall vibration. According to Biot's theory two longitudinal waves can propagate at the same time in both phases. The one mainly supported by the frame is called the frame-borne wave and the one mainly supported by the saturating fluid is called the airborne wave. ${ }^{4}$ A rotational wave may also be supported by the frame but it is not excited in the case of a onedimensional problem. The motion of the poroelastic media is described by the macroscopic displacement of the solid and fluid phases represented by the scalars $u^{s}$ and $u^{f}$, respectively. Four waves contribute to the displacement of one fluid or solid particle: two waves propagating in the $z$ direction and two waves propagating in the $-z$ direction [see Fig. 2(b)]. Since the transfer impedance will be applied to a piston radiating in an infinite half space, we consider only one acoustic wave propagating from the porous interface to the semi-infinite acoustic domain. This assumption allows us to use the simple relation between acoustic pressure and particle velocity on the porous surface: $p(0) / v(0)=Z_{0}$, where $Z_{0}$ is the characteristic impedance of the acoustic fluid.

The boundary conditions at the fluid-porous interface $(z=0)$ are: continuity of the normal stress in the solid and fluid phases with the external pressure

$$
\begin{aligned}
& \sigma_{z}^{s}(0)=-(1-\phi) p(0), \\
& \sigma^{f}(0)=-\phi p(0),
\end{aligned}
$$

where $\phi$ is the porosity of the porous material, and continuity of the total flow

$$
j \omega\left[(1-\phi) u^{s}(0)+\phi u^{f}(0)\right]=v(0) .
$$

At $z=-L$ the velocity of the fluid and the velocity of the frame are both equal to the wall velocity

$$
j \omega u^{s}(-L)=j \omega u^{f}(-L)=v_{p} .
$$

From Eqs. (7)-(10), it is possible to calculate amplitude of the five waves and derive the transfer impedance.

The surface impedance $Z_{s}$ is derived using the same formalism with the appropriate boundary conditions. In this configuration, an acoustic plane wave hit the porous layer at $z=0$ and Eqs. (7)-(9) are used to express the continuity of stress and total flow at this interface. On the surface of the porous layer in contact with the backing, the displacement of the air and the frame are equal to zero

$$
u^{s}(-L)=u^{f}(-L)=0 .
$$

The impedances $Z_{s}$ and $Z_{t}$ are derived for two kinds of porous materials, the characteristics of which are listed in Table I. Material A is a foam layer with a stiff skeleton and material B is a light fibrous layer with a very soft skeleton, both fairly resistive to airflow. Figures 3 and 4 show the simulation of the transfer impedance $Z_{t}$ and the surface impedance $Z_{s}$ for both materials.

When the wall is covered with material A (Fig. 3), it appears that the impedances $Z_{t}$ and $Z_{s}$ are similar as far as
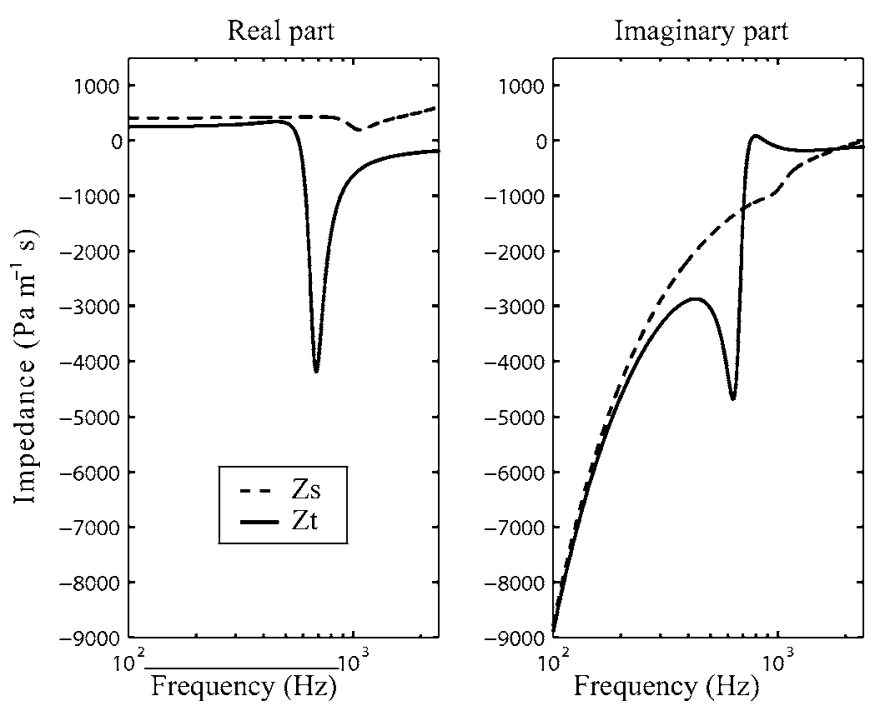

FIG. 3. Material A: real and imaginary part of the (- -) surface impedance $Z_{s}$ and (-) transfer impedance $Z_{t}$. 

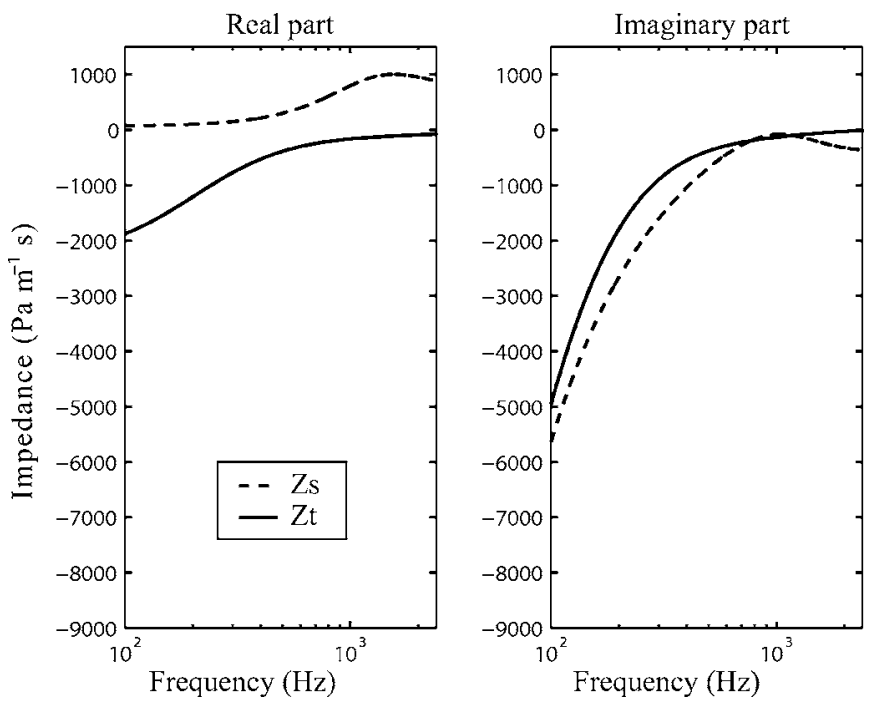

FIG. 4. Material B: real and imaginary part of the (- -) surface impedance $Z_{s}$ and $(-)$ transfer impedance $Z_{t}$.

$500 \mathrm{~Hz}$. Above this frequency, the real part of the transfer impedance becomes negative. Now, if the wall is covered with material B (Fig. 4), the imaginary part of both impedances is seen to be similar in the entire frequency range. However, the real parts are completely different, the real part of the transfer impedance being negative in the whole frequency range.

\section{Discussion}

For a better understanding, the vibratory behavior of the porous layers (A and B) is investigated for both kinds of excitation (depicted in Fig. 2).

Let us first consider the case of material A attached to the moving wall. Figure 5(a) (solid curve) shows the velocity ratio between the frame velocity at the air-porous interface and the wall velocity $v_{p}$ at $z=-L$. In the low frequency range, the velocity ratio is equal to one $(0 \mathrm{~dB})$, i.e., the material follows the wall movement and its thickness remains con- stant. At higher frequencies (up to $500 \mathrm{~Hz}$ ), the thickness of the porous layer does not remain constant during excitation and the velocity ratio reaches a maximum around $1100 \mathrm{~Hz}$. Figure 6(a) shows that this maximum appears around the first quarter-wavelength resonance frequency $f_{r}$ corresponding to the frame-borne wave: the Biot frame-borne wavelength is four times the layer thickness $L$. This resonance frequency may be approached by simply considering the properties of the frame in vacuo ${ }^{16}$ :

$$
f_{r} \approx \frac{1}{4 L} \sqrt{\frac{E \frac{(1-\nu)}{(1+\nu)(1-2 \nu)}}{\rho_{1}}},
$$

where $L$ is the thickness, $E, \nu$, and $\rho_{1}$ are, respectively, the Young modulus, the Poisson ratio and the density. For the given parameters (see Table I), $f_{r}$ is evaluated at $1047 \mathrm{~Hz}$ for material $\mathrm{A}$ and it is observed at around $1100 \mathrm{~Hz}$ in Fig. 6(a). Considering next the static wall configuration, Fig. 5(a) (dashed curve) shows the ratio between the frame velocity at the air-porous interface and the velocity of the acoustic fluid $v(0)$ at $z=0$. The low velocity ratio amplitude in the entire frequency band shows that the frame of the layer can be considered motionless, even at the resonance frequency of the frame. From the observations carried out in the low frequency range, it is possible to conclude that the acoustic behavior of material A, which is due to the fluid movement in the pores, is similar for both configurations because there is negligible deformation in the thickness of the layer. The frame's thickness tends to be constant at low frequencies because the wavelength of the frame-borne wave is long compared to the thickness of the layer as seen in Fig. 6(a). This explains the similarities observed below $500 \mathrm{~Hz}$ between the impedances $Z_{s}$ and $Z_{t}$. However, at higher frequencies, the vibratory behavior of the porous frame exerts a greater influence on the acoustical behavior of the layer and it is different for both configurations: the frame of the layer is excited much more by mechanical loading than by acoustical loading.
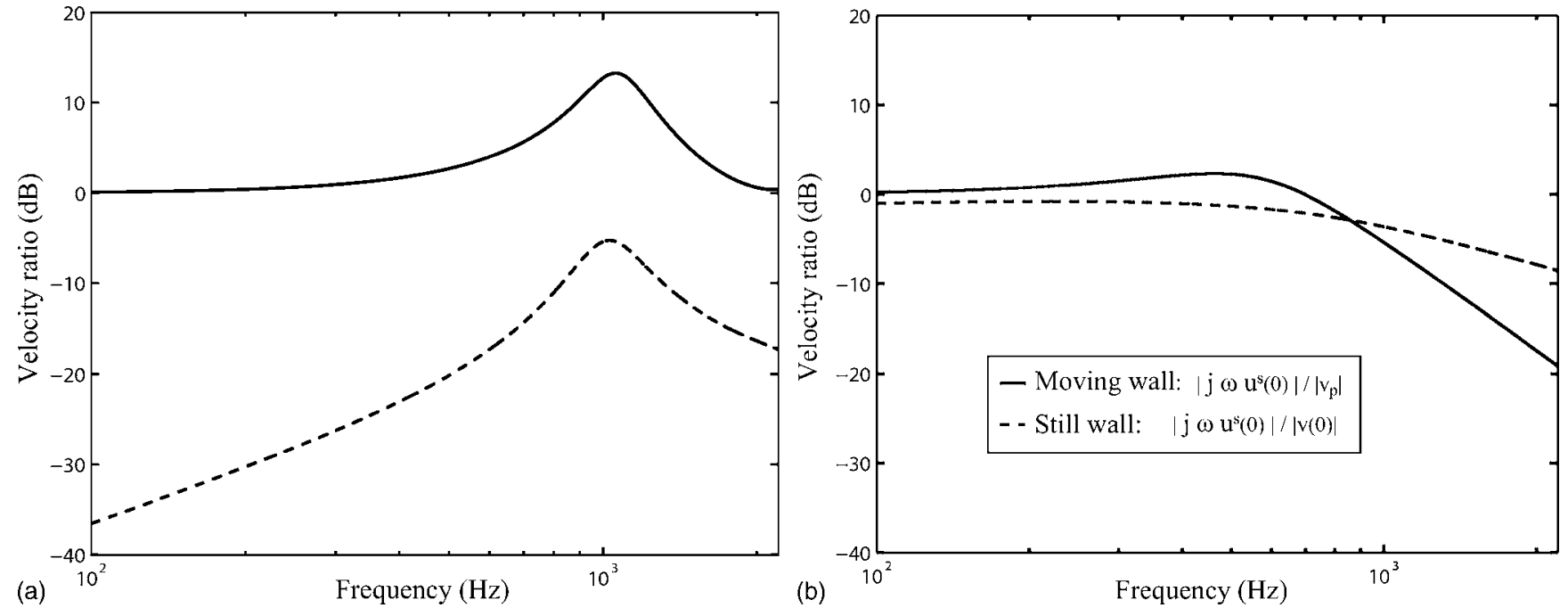

FIG. 5. Ratio between the velocity of the frame at the air-porous interface and: (- -) the velocity of the fluid at the porous surface $v(0)$ (still wall model), (-) the velocity of the piston $v_{p}$ (moving wall model): (a) Material A; (b) Material B. 

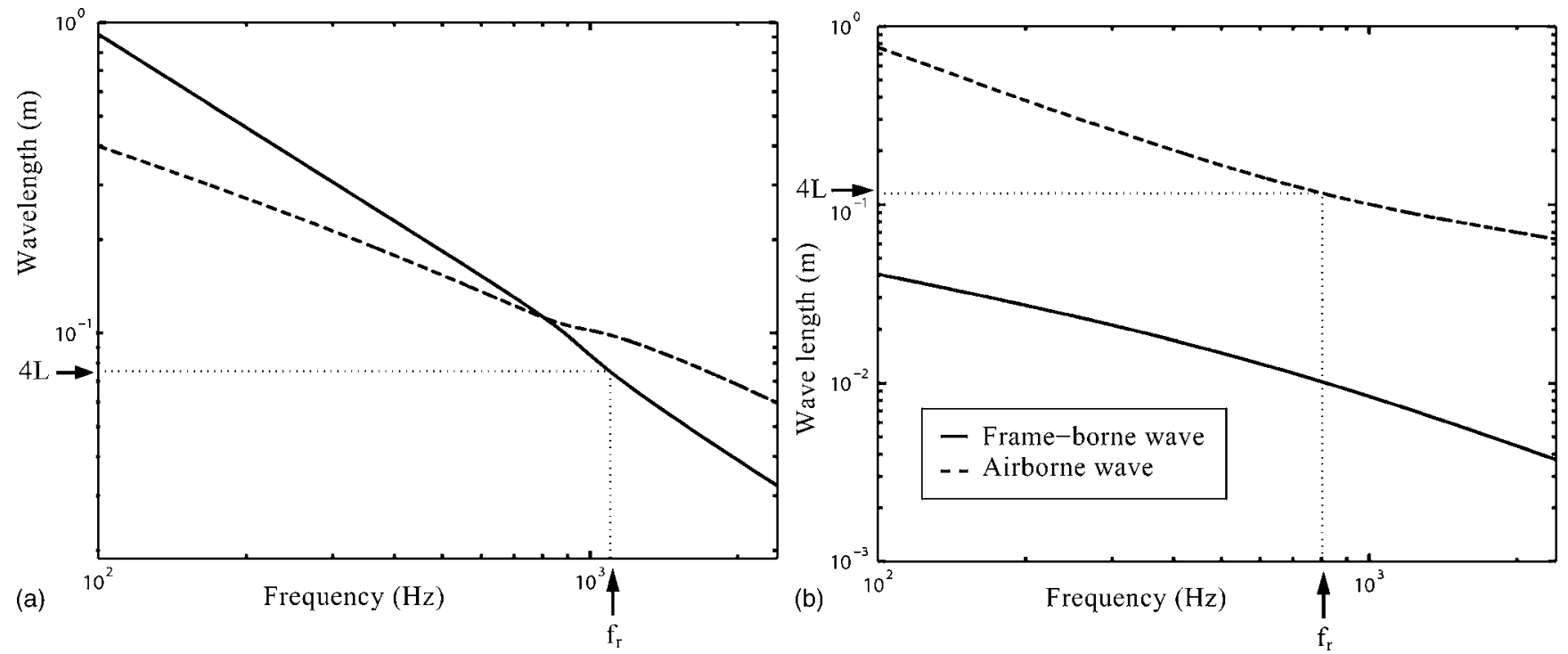

FIG. 6. Biot wavelengths: (a) Material A; (b) Material B.

Let us now consider the configuration in which the wall is covered with material B. If the source is the wall vibration [Fig. 5(b) solid curve], it is seen that the frame follows the wall movement at low frequencies. In the mid frequency range, a slight increase in the velocity ratio is observed, related in this case to the first $\lambda / 4$ resonance of the airborne wave [see Fig. 6(b)]. At higher frequencies, the velocity ratio decreases until it reaches $-20 \mathrm{~dB}$ at $2400 \mathrm{~Hz}$ which means that the frame tends to become motionless at the surface of the layer. Thus, material B allows a better decoupling between the vibration of the wall and the external media than material A because the relative velocity between both phases is greater. In the still wall configuration [Fig. 5(b) dashed curve] at low frequencies, it is seen that the ratio of the velocity of the frame at the air-porous interface to the velocity of fluid particle is close to $0 \mathrm{~dB}$. This means that the airflow drags the fibers and induces a deformation in the thickness of the fibrous layer. At higher frequencies, the velocity ratio decreases and the frame also tends to become motionless, but at a slower rate than in the case of the moving wall.

To sum up, the surface impedance $Z_{s}$ is close to the transfer impedance $Z_{t}$ in a restrictive frequency range where the frame can be considered "rigid" for both static and moving backing configurations. This occurs when the frameborne wavelength is greater than the porous layer thickness. In this paper, this only applies to material A below $500 \mathrm{~Hz}$.

\section{ACOUSTIC RADIATION OF A COVERED PISTON}

In order to compare simulations with experimental results, the acoustic radiation of a circular piston covered with a porous layer and set in an infinite baffle is investigated. The aim is to illustrate the consequences of using the surface impedance $Z_{s}$ instead of the transfer impedance $Z_{t}$ in an inhomogeneous mixed boundary condition.

\section{A. Theory}

The radiation efficiency factor $\sigma_{R}$ is used to characterize the acoustical effect of the covering. It is defined as the ratio of the radiated acoustic power, denoted as $W$, over the vibratory power

$$
\sigma_{R}=\frac{W}{\rho_{0} c_{0} S_{P} \frac{\left|v_{p}\right|^{2}}{2}},
$$

where $S_{P}$ is the piston surface, $\rho_{0}$ and $c_{0}$ are the ambient density and velocity of sound in air, and $v_{p}$ is the amplitude of the piston velocity. The radiated acoustic power $W$ can be derived by integrating the far field acoustic intensity $I(\boldsymbol{r})$ expressed in spherical coordinates $(r, \theta, \varphi)$ over a hemisphere of radius $r$,

$$
W=\int_{0}^{2 \pi} \int_{0}^{\pi / 2} I(\boldsymbol{r}) r^{2} \sin \theta d \theta d \varphi
$$

Calculus of the intensity uses the asymptotic form of the far field pressure $p(\boldsymbol{r})$ as:

$$
I=\frac{|p(\boldsymbol{r})|^{2}}{2 \rho_{0} c_{0}} .
$$

Mangulis ${ }^{8,9}$ and Feit and Duncan ${ }^{17}$ have investigated the pressure radiated from a rigid piston set in a nonrigid baffle. A similar approach is presented in the following. However, since we are interested in the influence of the covering applied to a moving structure, the baffle is taken as being perfectly reflecting.

Let us consider a circular piston in the infinite rigid baffle at $z=0$ (see Fig. 7). The surface of the piston is denoted by $S_{P}$, and the surface of the baffle by $S_{B}$. In the acoustic domain, the pressure $p$ is a solution of the harmonic Helmholtz equation [Eq. (3)]. The boundary conditions are: Sommerfeld condition 


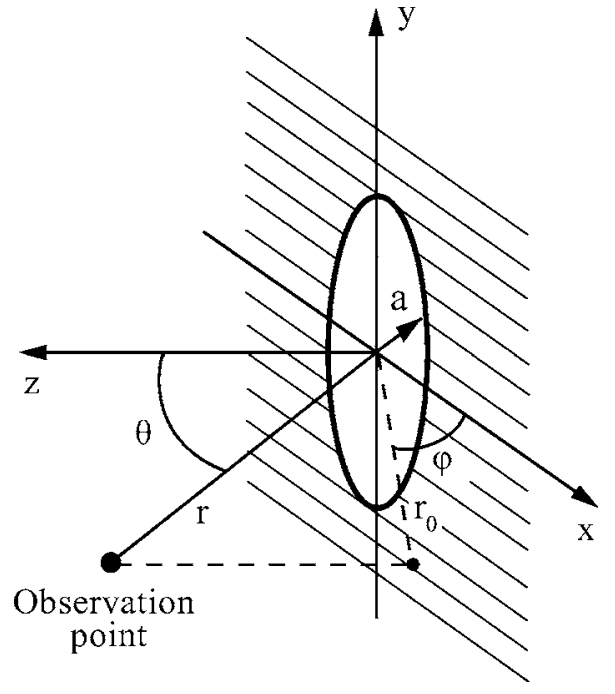

FIG. 7. Geometry of system and coordinates defining observation point.

$$
\lim _{r \rightarrow \infty} r\left(\frac{\partial p}{\partial r}+j k p\right)=0
$$

homogeneous Neumann condition on $S_{B}$

$$
\frac{\partial p}{\partial z}=0,
$$

and inhomogeneous mixed condition on $S_{P}$

$$
-\frac{\partial p}{\partial z}+j k \beta_{t} p=j \omega \rho_{0} v_{p}
$$

where $\beta_{t}=\rho_{0} c_{0} / Z_{t}$ and $Z_{t}$ is the transfer impedance of Eq. (1) expressed on the surface of the covering.

The pressure field is derived using the surface integral equation. The Green function (at $z=0$ ) chosen to satisfy the Sommerfeld boundary condition and the boundary condition

$$
\frac{\partial G}{\partial z}=0
$$

on both $S_{B}$ and $S_{P}$ is

$$
G\left(\boldsymbol{r}, \boldsymbol{r}_{0}\right)=\frac{e^{-j k\left|\boldsymbol{r}-\boldsymbol{r}_{0}\right|}}{2 \pi\left|\boldsymbol{r}-\boldsymbol{r}_{0}\right|} .
$$

Next, the solution of Eq. (3) subjected to the specified boundary conditions is given by

$$
p(\boldsymbol{r})=j \omega \rho_{0} \int_{S_{P}} v\left(\boldsymbol{r}_{0}\right) G\left(\boldsymbol{r}, \boldsymbol{r}_{0}\right) d S_{\mathbf{P}},
$$

where $v$ is the fluid velocity at the fluid-porous interface. This velocity $v$ can be expressed in terms of the piston velocity $v_{p}$ using the boundary condition of Eq. (18). First, the normal derivative of the acoustic pressure is replaced by the particle velocity using Eq. (4):

$$
j \omega \rho_{0} v+j k \beta_{t} p=j \omega \rho_{0} v_{p} .
$$

Then, a radiation condition at the fluid-porous interface is used to write the acoustic pressure in terms of the particle velocity, $Z_{\mathrm{rad}}=p / v$. Note that, since the plane wave ap-

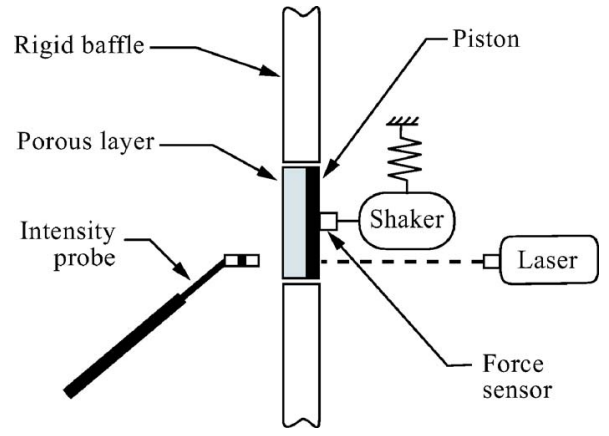

FIG. 8. Experimental setup schema to measure structure radiation efficiency.

proximation is used in the porous model to describe wave propagation (see Sec. II B), the acoustic pressure is related to fluid velocity by the impedance of the acoustic fluid $Z_{0}$. Furthermore, by definition, piston velocity $v_{p}$ is uniform on $S_{p}$. form:

Equation (21) can then be rewritten in the following

$$
p(\boldsymbol{r})=j \omega \rho_{0} \frac{Z_{t}}{Z_{t}+Z_{\mathrm{rad}}} \int_{S_{P}} v_{p} G\left(\boldsymbol{r}, \boldsymbol{r}_{0}\right) d S_{\mathbf{P}}
$$

This gives the relation between the far field pressure radiated by the bare system $p^{\prime}(\boldsymbol{r})$ and by the covered system $p(\boldsymbol{r})$,

$$
p(\boldsymbol{r})=\frac{Z_{t}}{Z_{t}+Z_{\mathrm{rad}}} p^{\prime}(\boldsymbol{r})
$$

where $p^{\prime}(\boldsymbol{r})$ is given by Morse and Ingard ${ }^{7}$ for a circular piston of radius $a$

$$
p^{\prime}(\boldsymbol{r})=j k \rho_{0} c_{0} v_{p} \frac{e^{-j k r}}{2 \pi r} \pi a^{2} \frac{2 J_{1}(k a \sin \theta)}{k a \sin \theta} .
$$

The multiplicative term of Eq. (24) has no influence on the far field directivity pattern. It acts mainly as an harmonic magnitude correction due to the presence of the covering.

\section{B. Experimental validation}

Radiation efficiency measurements of a piston with and without covering were performed. The flat piston was set in a rigid baffle (see Fig. 8) and radiated in an anechoïc room. The excitation was performed using a shaker driven with a white noise signal. The piston is honeycomb plate with a radius of $75 \mathrm{~mm}$. Because this structure is extremely stiff and light, its first natural frequency resonance are shifted above $2500 \mathrm{~Hz}$. Two types of covering materials were tested: a stiff foam layer $18.9 \mathrm{~mm}$ thick and a very soft fibrous layer $29 \mathrm{~mm}$ thick. The properties of these materials are given in Table I (see Sec. II B). The viscoelastic characteristics of the porous frames, Young's modulus $E$ and loss factor $\eta$, were measured at low frequency using the quasi-static method. ${ }^{18,19}$

The vibratory power of the plate was determined from the quadratic normal velocity measured at the piston surface by a laser vibrometer. The acoustic radiated power was determined from intensity measurements on porous surface using an intensity probe made from two $1 / 2$ in. microphones 

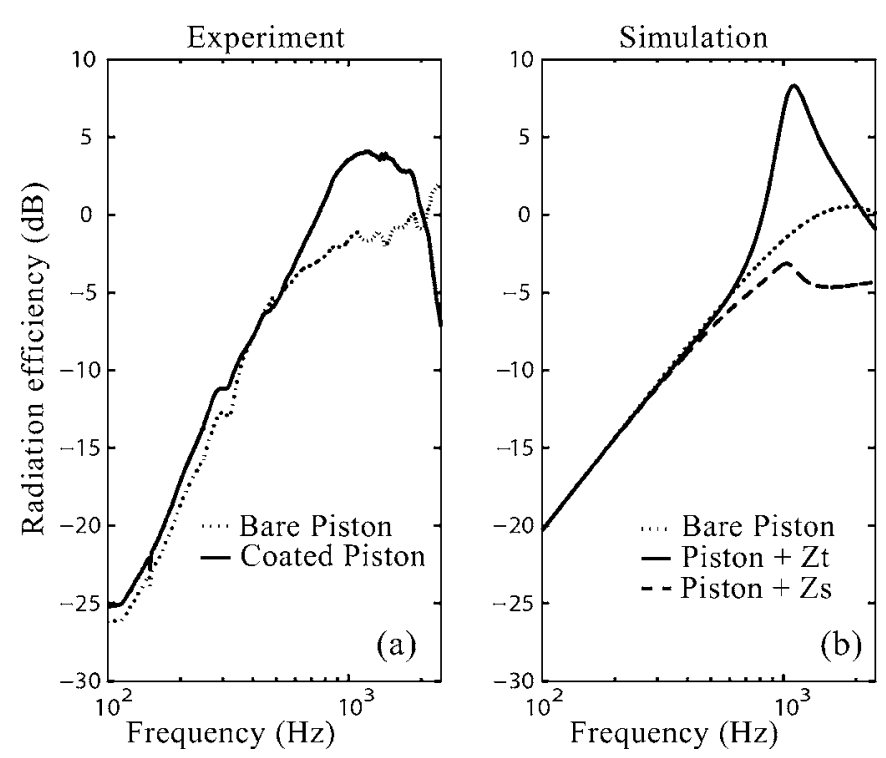

FIG. 9. Acoustic radiation efficiency of a bare and covered (material A) circular piston set in a rigid baffle: (a) experiment; (b) simulation.

$12 \mathrm{~mm}$ apart. The lateral dimensions of the piston are large enough in relation to the thickness of the porous layer for the effect of the edges to be ignored.

Figure 9(a) shows the radiation efficiency measurement of the bare piston and the radiation efficiency measurement of the piston covered with material A. Three distinct zones are observed. In the low frequency range, the porous layer has little or no effect on the radiation efficiency. In the mid frequency range, an amplification of the acoustic radiation is observed around the first resonance frequency of the frame $f_{r}$ (Eq. (12)). This phenomenon which deteriorates the insulation efficiency of the covering has already been observed by Harrison et al. ${ }^{20}$ in the case of vibration reduction using insulating mounts. Above $2000 \mathrm{~Hz}$, the radiation efficiency decreases due to losses and frame decoupling.

Figure 9(b) shows the simulated data when both the transfer impedance $Z_{t}$, which describes properly the moving boundary condition, and the surface impedance $Z_{S}$ (see Sec. II A) are applied in the inhomogeneous mixed boundary condition [Eq. (18)]. There is seen to be close agreement between the model including transfer impedance $Z_{t}$ (solid line) and the measurements over the entire frequency range considered, i.e., the radiation peak is predicted and a decrease is observed at higher frequencies. Note that the predicted radiation peak due to the frame resonance is sharper than the experimental one because the porous model only accounts for dissipations in one direction in the thickness of the porous layer. Furthermore, constant viscoelastic properties $E$ and $\eta$ measured at $5 \mathrm{~Hz}$ are used in the model, but are expected to increase at higher frequency. ${ }^{18,21,22}$ Thus, with more appropriate values of viscoelastic parameters, the simulated peak would be higher in frequency and lower in amplitude.

Figure 10(a) shows the radiation efficiency measurement of the piston covered with material B. It is seen that the same phenomena observed experimentally with material A occur with material B. An increase in radiation occurs around $800 \mathrm{~Hz}$ due, in this case, to the $\lambda / 4$ airborne wave resonance
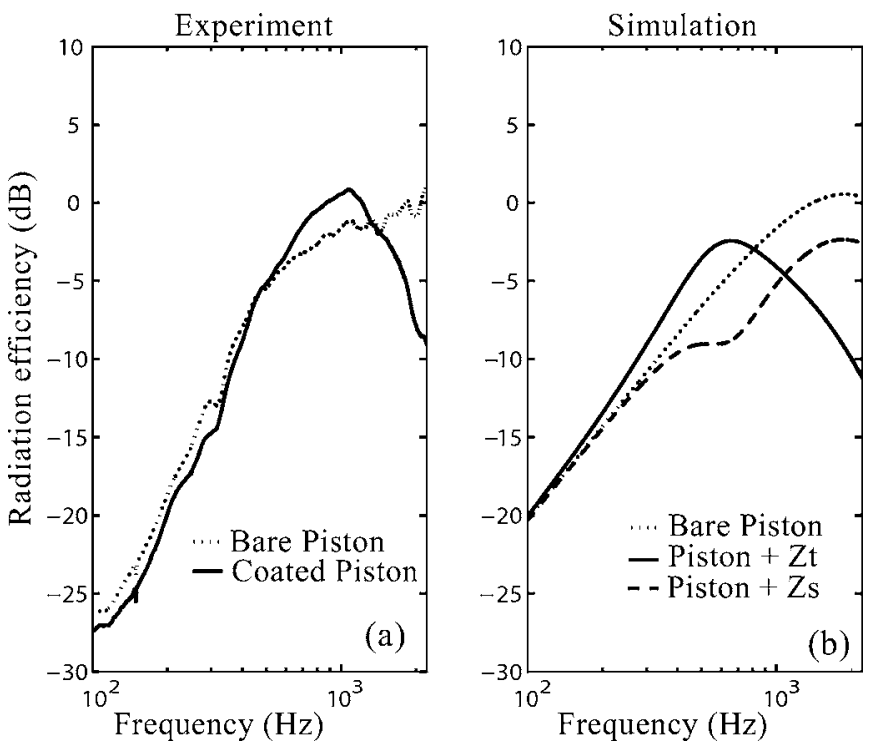

FIG. 10. Acoustic radiation efficiency of a bare and covered (material B) circular piston set in a rigid baffle: (a) experiment; (b) simulation.

in the thickness of the layer. At higher frequencies, the radiation efficiency strongly decreases compared to the radiation of the bare piston. The soft material B allows a better decoupling between the piston and the external media than material A. Note that only the model including the impedance $Z_{t}$ closely agrees with experimental data [see Fig. 10(b)].

In the frequency range where the covering has a significant effect, using $Z_{s}$ instead of $Z_{t}$ is improper: the radiation peak is not predicted and the high frequency drop does not agree with the experimental data.

\section{CONCLUSION}

In this paper, the impedance to be applied to a moving structure covered with a porous layer has been investigated. The well known surface impedance $Z_{s}$ which can easily be measured in an impedance tube is often applied to vibrating structures to account for the covering effects. However, it is shown that the impedance must be expressed in terms of the base movement to account for the real behavior of the layer. This impedance, called transfer impedance and denoted as $Z_{t}$, is derived using Biot's theory and compared to the surface impedance. It is shown that these impedances are similar as long as the longitudinal wavelengths are large compared to the layer thickness. In the case of a covered piston set in an infinite rigid baffle, the acoustic radiation peak and the decrease at higher frequencies due to the presence of the porous layer can only be predicted using the transfer impedance: the surface impedance measured in an impedance tube is not suitable in this case.

\section{ACKNOWLEDGMENTS}

The authors are grateful to the CTTM (Centre de Transfert de Technologie du Mans) where the measurements were performed and the CNRS and Région Pays de la Loire for 
their financial support. The authors would also like to thank Professor Michel Bruneau for his useful comments on the manuscript.

${ }^{1}$ G. Maidanik, "Use of decoupling to reduce the radiated noise generated by panels," J. Sound Vib. 81(2), 165-185 (1982).

${ }^{2}$ B. Laulagnet and J. L. Guyader, "Sound radiation from finite cylindrical coated shells, by means of asymptotic expansion of the three-dimensional equations for coating," J. Acoust. Soc. Am. 96(1), 277-286 (1994).

${ }^{3}$ A. Berry, O. Foin, and J. P. Szabo, "Three-dimensional elasticity model for a decoupling coating on a rectangular plate immersed in a heavy fluid," J. Acoust. Soc. Am. 109(6), 2704-2714 (2001).

${ }^{4}$ J. F. Allard, Propagation of Sound in Porous Media: Modelling Sound Absorbing Materials (Elsevier Applied Science, London, 1993).

${ }^{5}$ B. Laulagnet and J. L. Guyader, "Sound radiation from finite cylindrical coated shells covered with a compliant layer," Trans. ASME, J. Vib. Acoust. 113, 267-272 (1991)

${ }^{6}$ O. Foin, A. Berry, and J. P. Szabo, "Acoustic radiation from an elastic baffled rectangular plate covered by a decoupling coating and immersed in a heavy acoustic fluid," J. Acoust. Soc. Am. 107(5), 2501-2510 (2000).

${ }^{7}$ P. M. Morse and K. U. Ingard, Theoretical Acoustics (McGraw-Hill, New York, 1968).

${ }^{8} \mathrm{~V}$. Mangulis, "Radiation of sound from a circular rigid piston in a nonrigid baffle,” Int. J. Engng. Sci. 2(1), 115-126 (1964).

${ }^{9} \mathrm{~V}$. Mangulis, "Comments on: Numerical evaluation of the radiation impedance for a piston in a nonrigid baffle," J. Acoust. Soc. Am. 45, 10451046 (1969).

${ }^{10}$ S. Suzuki, S. Maruyama, and H. Ido, "Boundary element analysis of cavity noise problems with complicated boundary conditions," J. Sound Vib. 130(1), 79-91 (1989).

${ }^{11}$ A. F. Seybert and D. F. Ross, "Experimental determination of acoustic properties using a two-microphone random-excitation technique," J. Acoust. Soc. Am. 61, 1362-1370 (1977).
${ }^{12}$ A. Piot, J. M. Ville, and J. M. Auger, "Rayonnement acoustique dans un conduit par un piston recouvert d'un matriau absorbant," Proc. of the International Symposium on the Prediction of the Noise Emitted by Vibrating Structures, CETIM-Senlis, March 26-28 (1991).

${ }^{13}$ W. Shao and C. K. Mechefske, "Analysis of the sound field in finite length infinite baffled cylindrical ducts with vibrating walls of finite impedance," J. Acoust. Soc. Am. 117(4), 1728-1736 (2005).

${ }^{14} \mathrm{M}$. A. Biot, "The theory of propagation of elastic waves in a fluidsaturated porous solid. I. Low frequency range. II. Higher frequency range," J. Acoust. Soc. Am. 28, 168-191 (1956).

${ }^{15}$ B. Brouard, D. Lafarge, and J. F. Allard, "A general method of modelling sound propagation in layered media," J. Sound Vib. 183(1), 129-142 (1995).

${ }^{16}$ J. F. Allard, C. Depollier, P. Guignouard, and P. Rebillard, "Effects of a resonance of the frame on the surface impedance of glass wool of high density and stiffness," J. Acoust. Soc. Am. 89, 999-1001 (1991).

${ }^{17}$ D. Feit and M. E. Duncan, "Numerical evaluation of the radiation impedance for a piston in a nonrigid baffle," J. Acoust. Soc. Am. 43, 885-886 (1968).

${ }^{18}$ N. Dauchez, M. Etchessahar, and S. Sahraoui, "On measurement of mechanical properties of sound absorbing materials," Second Biot Conference on Poromechanics, Grenoble, France, Sept. 14-16 (2002), pp. 197-202.

${ }^{19}$ M. Etchessahar, S. Sahraoui, L. Benyahia, and J. F. Tassin, "Frequency dependence of elastic properties of acoustic foams," J. Acoust. Soc. Am. 117(3), 1114-1121 (2005)

${ }^{20} \mathrm{M}$. Harrison, A. O. Sykes, and M. Martin, "Wave effects in isolation mounts," J. Acoust. Soc. Am. 24, 62-71 (1952).

${ }^{21}$ T. Pritz, "Unbounded complex modulus of viscoelastic materials and the Kramers-Kronig relations," J. Sound Vib. 279, 687-697 (2005).

${ }^{22}$ O. Doutres and N. Dauchez, "Characterization of porous materials viscoelastic properties involving the vibroacoustical behavior of coated panels," Proc. of the International Symposium on the Acoustics of PoroElastic Materials (SAPEM), Lyon, France, Dec. 7-9 (2005). 